\title{
Forgetting of a simple motor task
}

WAYNE L. FOX ${ }^{2}$ AHD CECIL A. ROGERS, JR. UNIVERSITY OF ARIZONA

Severe motor-skills forgetting was shown in a simple printing task. Decrements were attributed in large part to retroactive interference produced via a training procedure previously proposed by Rogers (1966). Interpretation was in terms of conflicting sets. Inadequacies of traditional indices of motor forgetting were emphasized.

Research involving the investigation of perceptualmotor skills retention has been reviewed extensively yielding the same general conclusions: (1) little forgetting occurs, and (2) interference effects, when found, are small and transitory (McGeoch \& Irion, 1952; Bilodeau \& Bilodeau, 1961; Adams, 1964; Irion, 1966). Previous research has included recall intervals from a few minutes to several years; tasks have varied in complexity from lever positioning to multi-dimensional tracking. In spite of failures to produce a substantial interference effect, there has been much systematic research. Perhaps Lewis and his associates have most thoroughly investigated the effect of retroactive interference (RI) on the retention of motor tasks (e.g., Lewis, 1959). These researchers employed the classical ABA paradigm wherein a second practice task was interpolated between an original task and its recall. The RI effects that were found were not substantial and tended to dissipate rapidly. The average amount of RI obtained in such tasks was less than 10\%; more than $30 \%$ has not been demonstrated.

Rogers (1966) has proposed the Incidental-Intentional Training Paradigm as a method for producing large amounts of RI. The design includes practice of an incidental task which is to be recalled after performance of an intentional task. Rogers reported RI in excess of $50 \%$ with total number of interfering responses in excess of $80 \%$, for the recall of meaningful verbal materials. These effects were ascribed to two variables: (1) inadequate or conflicting sets, and (2) an impoverished recall environment. That interpretation was consistent with McGeoch \& Irion (1952) who also emphasized inadequate set at recall as a major prerequisite for forgetting.

The present study applied Rogers' paradigm to a motor-skills task in an attempt to produce substantial RI. The production of inadequate set was accomplished by instructing $S s$ to practice drawing a specified geometric figure as accurately and precisely as possible. The result was a relatively slow self-paced response rate. Ss were then instructed to draw the figure as rapidly as they could, which yielded a relatively fast response rate. During recall, Ss were requested to draw the figure at the same rate as they had previously during the first practice period when accuracy had been stressed by $E$. Under these conditions $S$ would be expected to enter the recall period with two conflicting sets: one to respond accurately and one to respond rapidly. It was expected that sufficient RI would obtain that rate of responding would increase substantially when compared to a control group which had not performed the intervening speed task. The net result would be severe forgetting.

\section{Method}

Ss were 311 introductory psychology students at the University of Arizona unsystematically divided into two groups, an RI group $(\mathrm{N}=153)$ and a control group $(\mathrm{N}=$ 158). All Ss were tested simultaneously at their second class meeting.

Self-paced rate of reproduction of a geometric figure (the upper case Greek letter Phi) was the training task used. The specific response measure was the number of test figures drawn per one minute interval.

All Ss were provided with pencils and seven-page test booklets with alternate instruction and response pages. Ss were treated as follows: first, all Ss were instructed to practice drawing the figure as accurately as possible (60 sec. accuracy trial); secondly, the RI group was instructed to draw the figure as rapidly as possible (60 sec. speed trial) while the control group studied a short prose passage (60 sec. control task); finally, both groups were instructed to recall the rate at which they had first practiced drawing the figure accurately ( $60 \mathrm{sec}$. recall trial). To extend the retention interval, all Ss were required to study a list of nonsense syllables during the interval prior to recall $(120 \mathrm{sec}$. interpolated task). Since the time allotted for each instruction page was 20 sec., the total recall interval was $240 \mathrm{sec}$. The general design is summarized below:

$\begin{array}{lccll}\text { Group } & \begin{array}{c}\text { Incidental } \\ \text { Task }\end{array} & \begin{array}{c}\text { Intentional } \\ \text { Task }\end{array} & \begin{array}{c}\text { Interpolated } \\ \text { Task }\end{array} & \text { Recall } \\ \text { RI } & \begin{array}{c}\text { Accuracy } \\ \text { Trial }\end{array} & \begin{array}{c}\text { Speed } \\ \text { Trial }\end{array} & \begin{array}{c}\text { Nonsense } \\ \text { Syllables }\end{array} & \begin{array}{c}\text { Respon } \\ \text { Rate of } \\ \text { Accuracy }\end{array} \\ & & & & \\ & & & \\ \text { Control } & \text { Same } & \begin{array}{c}\text { Control } \\ \text { Task }\end{array} & \text { Same } & \text { Same }\end{array}$

\section{Results and Discussion}

Means and variances for both groups are presented in Table 1. It will be noted that $\mathrm{Ss}$ in both groups performed essentially the same during the first (incidental) training period. During recall, substantial forgetting was evidenced by a strong increase in rate of responding for all but three out of $311 \mathrm{Ss}$. This failure to recall the original response rate produced $t$ values of $18.88(152 \mathrm{df}$ ) for the RI group and 18.58 (157 df) for 
Table 1. Means and Variances for the Experimental and Control Groups During Training and Recall

\begin{tabular}{llccc} 
Group & & Practice Trial* & Speed Trial & Recall** \\
\hline \multirow{2}{*}{ RI } & $\bar{x}$ & 5.42 & 16.02 & 11.80 \\
& $\mathrm{~s}^{2}$ & 8.85 & 49.18 & 27.76 \\
\multirow{2}{*}{ Control } & $\mathrm{x}_{2}$ & 5.64 & -- & 9.22 \\
& $\mathrm{~s}^{2}$ & 9.49 & -- & 20.57 \\
\hline
\end{tabular}

${ }^{*} t=.64,309 \mathrm{df}, \mathrm{NS} ;{ }^{* *} t=7.01,309 \mathrm{df}, \mathrm{p}=.001$

the control group, indicating that the recall decrements were quite significant $(p<.001)$. This forgetting by both groups is ascribed to inadequate set at recall. Ss were trained to draw with accuracy but were required to recall response rate. Thus, the training task was incidental with respect to the rate response measure; if accuracy of response could be objectively measured, the resultant loss might have been minimal. Should this overall forgetting be due in fact to set, then such a loss should be immediate. Such was demonstrated by the subsequent testing of an additional group of $90 \mathrm{Ss}$, who were examined using a 20 sec. retention interval under otherwise identical procedures. Results paralleled those of the control group reported above with no significant differences between them.

Of more importance is the difference between RI and control groups $(t=7.01$ with 309 df, $p<.001)$. The intentional task or speed trial did produce strong additional interference in the recall of the original practice rate. Such a substantial amount of obtained RI argues against the thesis that proactive processes may account for all but a small percentage of what is forgotten (Underwood, 1957). As with many other sets of variables the relative contribution of proactive and retroactive processes may vary greatly with tasks, procedures, individuals, and other factors. It would then be unfortunate if motorskills retention, and $\mathrm{RI}$ in particular continue to lose interest as research topics, now that a more potent procedure is available for their investigation.

The data reported in the present study also indicate a major difficulty in the standard indices of forgetting and interference. The traditional formula $100 \cdot$ (TrainingRecall)/Training, yields a net loss for the RI group of $117.7 \%$, of which $78.2 \%$ is attributable directly to RI. In the past this computational procedure was more satisfactory, as traditional techniques offered little danger of producing over $100 \%$ forgetting. An alternate formula which utilizes the maximum possible number of responses that $S$ can produce in an allotted time interval is $100 \cdot$ (Training-Recall)/Maximum Possible
Response Decrement. This formula yields a total retention decrement of $60.2 \%$ in the RI group, of which $46.0 \%$ can be directly attributed to RI. Neither formula is entirely satisfactory for the data presented, but both are reported to allow the reader to estimate the potency of the obtained RI effect.

One major difficulty involved in the use of the paradigm lies in determining degree of incidentality of performance. In essence this problem resolves itself into one of S's division of his attention among the various aspects of his responding, both momentarily and the distribution of these divisions over training. As in the present task of printing, which draws upon a strong culturally-established habit, $S$ is not simply printing. He is drawing at a certain rate, with a given pressure, some degree of accuracy, a particular grip on the pencil, etc. E's instructions are at best a gross method of manipulating these attentional aspects which, in turn, govern performance relationships among tasks.

Motor forgetting must be demonstrated in the laboratory before the variables affecting it can be considered and it is a simple fact that more traditional techniques have not succeeded. The Incidental-Intentional Training Paradigm, however, is a procedure with broad implications for the study of such forgetting. Tasks such as card sorting, lever positioning, cranking, and tracking should be readily amenable to the operations involved in the paradigm.

\section{References}

Adams, J. A. Motor skills. Annu. Rev. Psychol., 1964, 15, 181-202. Bilodeau, E. A., \& Bilodeau, I. McD. Motor-skills learning. Annu. Rev. Psychol., 1961, 12, 243-280.

Irion, A. L. A brief history of research on the acquisition of skill. In E.. A. Bilodeau (Ed.), Acquisition of skill. New York: Academic Press, 1966.

Lewis, D. Latest news and views regarding individual susceptibility to interference in the performance of perceptual-motor tasks. Paper read at Midwestern Psychological Association meetings, Chicago, 1959.

McGeoch, J. A., \& Irion, A. L. The psychology of human learning. New York: Longmans, Green and Co., 1952.

Rogers, C. A., Jr. Retroactive interference as a function of set and recall environment. Psychon. Sci., 1966, 5, 223-224.

Underwood, B. J. Interference and forgetting. Psychol. Rev., 1957,. 64, 49-60.

\section{Notes}

1. This paper is partially based upon a dissertation written by the senior author and submitted to the Graduate College of the University of Arizona in partial fulfillment of the requirements for the Ph.D. degree. Research was supported in part by NASA Grant NGR-03-002-091.

2. Now at HumRRO, Training Center Human Research Unit, Presidio of Monterey, California. 